Running Head: Analysis of doping control tests from 2003 to 2015

\title{
Type: Review
}

Analysis of doping control test results in individual and team sports from 2003 to 2015

Authors: Millán Aguilar-Navarro ${ }^{1,2}$, Jesús Muñoz-Guerra ${ }^{3}$, María del Mar Plata ${ }^{4}$, Juan $\operatorname{Del~Coso}{ }^{1, *}$

${ }^{1}$ Exercise Physiology Laboratory, Camilo José Cela University, Madrid, 28692,Spain

2 Faculty of Education, Francisco de Vitoria University, Madrid, 28223, Spain

3 Department for Doping Control, Spanish Agency for Health Protection in Sport,

Madrid, 28016, Spain

4 Department of Education, Spanish Agency for Health Protection in Sport, Madrid, 28016, Spain

* Correspondening author.

E-mail: jdelcoso@ucjc.edu

Received: 2018-11-21

Revised: 2019-2-27

Accepted: 2019-4-2 


\section{Graphical Abstract}

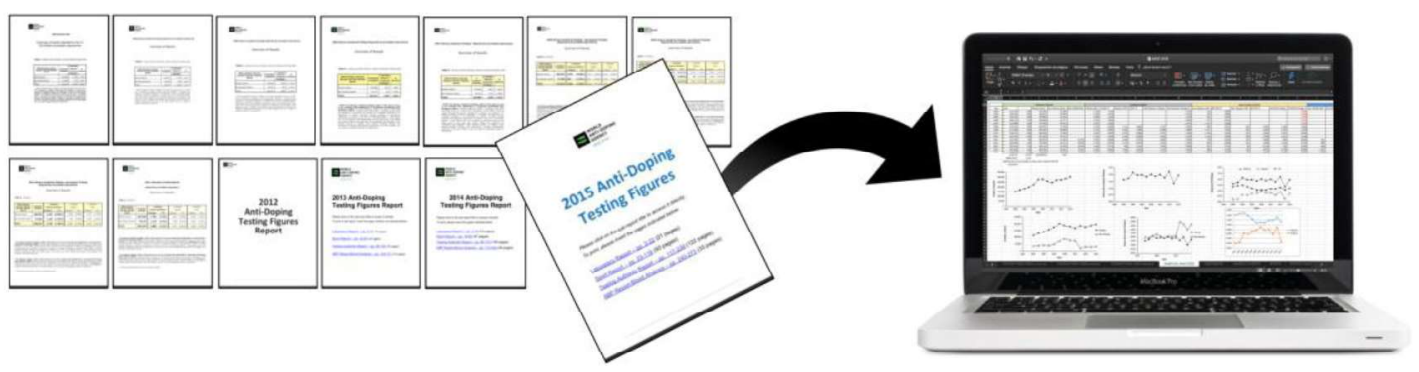

Sports with the highest proportion of adverse/atypical findings

Individual sports

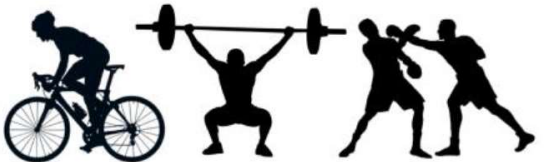

$3.3 \pm 1.0 \% \quad 3.0 \pm 0.6 \% \quad 2.9 \pm 0.6 \%$
Team sports

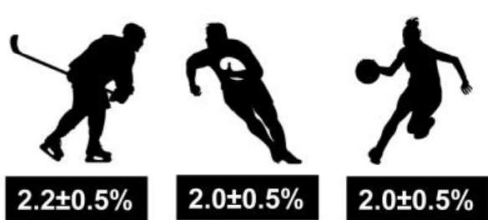

\section{ABSTRACT}

Background: Determining the prevalence of doping in sport might be useful for anti-doping authorities to gauge the effectiveness of anti-doping policies implemented to prevent positive attitudes toward doping. Using questionnaires and personal interviews, previous investigations have found that the prevalence of doping might be different among different sports disciplines; however, there is no sport-specific information about the proportion of adverse and atypical findings in samples used for doping control. The aim of the present investigation was to assess the differences in the frequency of adverse analytical and atypical findings among sports using the data made available by the World Anti-Doping Agency.

Method: The data included in this investigation were gathered from the Testing Figures Reports made available annually from 2003 to 2015 by WADA. These Testing Figures Reports include information about the number of samples analyzed, the number of adverse and atypical findings reported, and the most commonly found drugs in the urine and blood samples analyzed. A total of 
$1,347,213$ samples were analyzed from the individual sports selected for this investigation, and 698,371 samples were analyzed for disciplines catalogued as team sports.

Results: In individual sports, the highest proportions of adverse and atypical findings (AAF) were $3.3 \% \pm 1.0 \%$ in cycling, $3.0 \% \pm 0.6 \%$ in weightlifting and $2.9 \% \pm 0.6 \%$ in boxing. In team sports, the highest proportions of AAF were $2.2 \% \pm 0.5 \%$ in ice hockey, $2.0 \% \pm 0.5 \%$ in rugby and $2.0 \% \pm$ $0.5 \%$ in basketball. Gymnastics and skating had the lowest proportions $\leq 1.0 \%$ ) for individual sports, while field hockey, volleyball and football had the lowest proportions for team sports $(<1.5 \%)$.

Conclusion: As suggested by the analysis, the incidence of AAF was not uniform across all sports disciplines, with the different proportions pointing to an uneven use of banned substances depending on the sport. This information might be useful for increasing the strength and efficacy of anti-doping policies in those sports with the highest prevalence in the use of banned substances.

Keywords: Anti-doping, Attitude, Banned drugs, Elite athlete, Type of sport 


\section{Introduction}

Since the creation of the World Anti-Doping Agency (WADA) in 1999, there has been a strong anti-doping movement across sports organizations and governmental authorities, with the main objective being to reduce the prevalence and incidence of doping in sports. ${ }^{1}$ Although several important steps in this direction have been taken in the past 20 years, this objective is far from being fully achieved because at present the use of banned substances by elite athletes from different sports disciplines is a certainty. ${ }^{2-4}$ Some flaws in the current anti-doping system, including the low deterrent effect of the punishment established for athletes sanctioned for an anti-doping rule violation, the inclusion of substances on the banned list without proper scrutiny of their effects on physical performance, and the imperfections in the Therapeutic Use Exemption (TUE) protocol, may have contributed to the relatively low efficacy of anti-doping policies.

Determining the prevalence of doping in sport is important for all the entities involved in sports organization; however, assessment of the prevalence and the changes in the incidence of doping is particularly crucial for anti-doping authorities to gauge the effectiveness of their antidoping policies and the utility of the social and economic investment made to prevent positive attitudes toward doping. Even though it is impractical to identify the exact prevalence and incidence of doping in sport, various analyses have shown that intentional doping occurs in $14 \%-57 \%$ of the population of competitive athletes. ${ }^{6-9}$ Some of these investigations have used surveys based on the randomised response technique, which allows the athlete to maintain anonymity while answering questions about doping practices. However, the accuracy of these survey-based investigations is limited by the necessity for truthfulness, ${ }^{10}$ objectivity, and the necessity that athletes' have an accurate knowledge of what is a doping misconduct. ${ }^{11}$ According to the results of doping control tests, it has been revealed that the percentage of samples containing banned substances has remained relatively stable at $\sim 2.0 \%$ both before ${ }^{6}$ and after $^{12}$ the creation of WADA, a lower proportion than the one found in investigations based on personal input. ${ }^{11}$ The assessment of the prevalence and 
incidence of doping in sport using the results of doping tests is an objective and robust method, but it suffers from the limitations of the small detection window that exists for specific prohibited substances $^{13}$, the analytical capability of WADA-accredited laboratories at the time of analysis, and an inability to discern the legal use of prohibited substances for therapeutic purposes from the intentional use of banned substances to obtain a competitive advantage.

To date, most of the strategies implemented to fight against doping have failed to consider the particularities of each sport discipline in terms of doping misconduct (prevalence and seasonal variations in the use of banned substances, the substances consumed and methods most used to consume them, and so forth). Determining the prevalence of doping practices and recognizing the differing attitudes that exist toward doping in various sports can aid in the management and implementation of plans for doping control tests in each sports discipline. It is likely that the use of anti-doping policies specifically designed to address the characteristics of doping misconduct in each sport can improve the effectiveness of current anti-doping strategies. However, the information needed to understand the differences in the prevalence of and attitudes toward doping among various sports is scarce, making it difficult to draw conclusions about which anti-doping measures to use.

A few investigations have assessed the prevalence of doping in one or various sports, but the methods used to assess the prevalence of doping are diverse and hinder an objective comparison among sports. Doping in athletics, as measured by surveys, might be up to $57 \%{ }^{7}$ In addition, track and field athlete's nationality might be one of the major factors affecting prevalence of doping in this sport, ${ }^{14}$ suggesting the existence of contrasting attitudes toward doping between countries. Likewise, the incidence of doping may be high in cycling since the use of doping agents was admitted by a high proportion of cyclists that complated a questionnaire about the use of doping agents in the past 3 months. ${ }^{15}$ However, not all the investigations confirm this high prevalence of doping in cycling. ${ }^{16}$ In contrast, all the players in the 32 football teams classified for the Brazil 2014 World Cup were tested for doping control out of competition and there was not any case of intentional doping in the more 
than 1,000 samples analyzed. ${ }^{17}$ It has also been found ${ }^{18}$ that offers to use banned substances are received most often by speed and power athletes, followed by endurance athletes, then by participants in sports that require motor skills, and then by participants in team sports. However, athletes rarely admit the use of banned substances despite the ease with which they can obtain doping agents. ${ }^{18}$ These findings coincide with findings from other investigations that have reported that participants in individual sports were more prone to be involved in doping than participants in team sports. ${ }^{19}$ Furthermore, athletes practising individual sports that require motor skills are less likely to be involved in doping practices than athletes practising more physically-oriented individual sports. ${ }^{4}$ Finally, track and field athletes, cyclists and weightlifters had a higher knowledge of anti-doping rule violations than their counterparts in other Olympic sports. ${ }^{20}$ Taken together, previous research suggests that differences exist among various sports in the prevalence of doping and in athletes' attitudes toward doping.

Because data has been gathered for only a few sports, an objective comparison of the prevalence/incidence of doping in various sports disciplines has not been possible. Thus, the aim of the present investigation was to analyse the frequency of and atypical findings reported by WADAaccredited laboratories from 2003 to 2015 to assess the differences in the incidence of doping in various sports.

\section{Methods}

The data included in this investigation were gathered from the Testing Figures Reports made available annually from 2003 to 2015 by WADA. These Testing Figures Reports can be accessed from the WADA website, and they include information about the number of samples analyzed, the number of adverse and atypical findings(AAF) reported, and the most commonly found drugs in the urine and blood samples analyzed. ${ }^{21}$ The percentage of AAF was calculated for each sport as described below.To determine the presence of a prohibited substance or its main metabolites or 
markers in athletes participating in both Olympic and non-Olympic sports from 2003 to 2015, WADA-accredited laboratories analyzed a total of 3,103,974 samples. The current investigation presents an $a d$ hoc analysis of the samples for 17 individual sports (Aquatics, Athletics, Boxing, Canoe/Kayaking, Cycling, Fencing, Gymnastics, Judo, Rowing, Shooting, Skiing, Skating, Taekwondo, Tennis, Triathlon, Weightlifting and Wrestling) and 7 team sports (Basketball, Football, Handball, Field Hockey, Ice Hockey, Rugby and Volleyball). All these sports were part of the Olympic programme during the analyzed period. (Information on Rugby was available only from 2012 to 2015). For this investigation, only complex team sports were labelled as "team sports", while other individual disciplines that have collective events (known as simple and aggregated team sports, including athletics, swimming, cycling, rowing, etc ${ }^{22}$ remained labelled as individual sports because most of the samples analyzed came from the individual disciplines. The grouping of individual and team sports in the current analysis only responds to the intention of creating a more comprehensible manuscript, as some individual and team sports share physical, physiological and sociodemographic characteristics. Because it was impractical to analyze all the sports (more than 100) included in the WADA reports, the above-mentioned individual and team sports were selected because they accounted for at least 1,000 samples per year in all the years examined.

The Testing Figures Reports have evolved from 2003 to 2015 and have presented more information about doping every year. However, we have used the data from 2003 as the basis for the analysis. In the reports from 2003 to 2007,2 types of non-negative results were merged: a) adverse analytical findings (when the laboratory detected in the athlete's sample a substance and/or a marker and/or a metabolite of the substance that was included in the list of prohibited substances) and b) atypical findings (when the parameters measured by the laboratory showed a discrepancy with the previous results for the same athlete). Notably, the analyses did not include information about Therapeutic Use Exemption; thus, adverse and atypical findings did not necessarily end in antidoping rule violations. The information on the number of samples obtained in competition and out of 
competition and the number of AAF have been analyzed using the reports from 2012 to 2015 because this information was not included in the previous reports. Finally, the blood samples collected for the Athlete's Biological Passport were not included in our analysis because this type of sample had not been obtained during the entire period from 2003 to 2015 .

\subsection{Statistical analysis}

The data were electronically extracted from the Testing Figures Reports and entered into a database designed for the purposes of this research. The data were extracted by one author (MAN) using a spreadsheet (Excel 2016, Microsoft Office, WA, USA) and then they were checked for accuracy by another author (JDC). Subsequently, the mean \pm SD for each Olympic sport (individual or team) was calculated for span of years investigated $(2003-2015)$. The normality of each variable was initially analyzed with the Shapiro-Wilk test. A one-way analysis of variance (ANOVA) was used to detect differences in the number of samples analyzed and in the frequency of AAF among sports. Afer a F significant test (inter-groups and intra-groups degrees of freedom), the Bonferroni post hoc analysis was employed to identify differences among individual sports and among team sports in these variables. The differences in the distribution of samples and the percentage of AAF between in-competition and out-of-competition settings were tested with crosstab and Chi square tests, including adjusted standardised residuals. Briefly, a sport that had a distribution of samples or percentage of AAF was considered to be statistically different from expected when its distribution of samples/findings in-competition and out-of-competition was $>$ or $<$ than the critical value of $Z$ (i.e., 1.96). The data were analyzed with the statistical package SPSS version 20.0 (SPSS Inc., Chicago, IL, USA). The significance level for all tests was set at $p<0.05$.

\section{Results}


A total of 1,347,213 samples were analyzed from the individual sports selected for this investigation from 2003 to 2015, with an overall frequency of AAF of $1.6 \% \pm 0.9 \%$. In the team sports examined, the number of samples analyzed during this period was 698,371, with the overall frequency of $\operatorname{AAF}(1.7 \% \pm 0.6 \%)$ being similar to individual sports. Table 1 depicts the average number of samples analyzed per year in all individual sports $(F(16,204)=218.1 ; p($ ANOVA $)<$ 0.001). Athletics was the individual sport with the highest number of samples analyzed per year, with a significantly greater number of samples being available than the remaining individual sports $(p<$ 0.01 in all pairwise comparisons). Cycling ranked second among the second individual sport in the number of samples analyzed per year and had higher values for adverse and atypical findings than all the remaining individual sports ( $p<0.01$ in all pairwise comparisons). Aquatics and weightlifting also had a higher number of samples analyzed per year than the remaining individuals sports $(p<$ 0.01 in all pairwise comparisons). Table 1 contains the number of AAF per sport from 2003 to 2015 . In absolute values, cycling, athletics and weightlifting were the sports with the highest number of non-negative results. Table 1 also contains the average \pm SD in the proportion of adverse and atypical findings in individual sports, which eliminates the influence of the differences in the number of samples analyzed in each individual sport $(F(16,204)=24.6 ; p($ ANOVA $)<0.001)$. Cycling, weightlifting and boxing were the individual sports with the highest proportion of AAF, with the percentages being greater than the remaining individual sports ( $p<0.01$ in all pairwise comparisons). Aquatics, rowing, shooting, gymnastics, and skating had the lowest proportion of AAF in the years studied, and their values were also lower than the proportions of adverse and atypical findings found in triathlon and wrestling ( $p<0.01$ in all pairwise comparisons).

Table 1 presents information on the average number of samples analyzed $(F(6,84)=262.7$; $p($ ANOVA $)<0.001)$ in team sports. Football was the team sport with the highest number of samples analyzed per year, with a significantly greater number of samples than the remaining team sports $(p<$ 0.01 in all pairwise comparisons). Basketball ranked second in team sports in the number of samples 
analyzed per year, with a significantly greater number of samples than volleyball, ice hockey, handball and field hockey ( $p<0.01$ in all pairwise comparisons). Football, basketball and rugby were the team sports with the highest number of AAF. However, ice hockey and rugby were the team sports with the highest proportion of $\operatorname{AAF}(F(6,84)=8.1 ; p$ (ANOVA) $<0.001)$, with higher proportions than field hockey, volleyball and football ( $p<0.05$ in all pairwise comparisons). Basketball also had a higher proportion of adverse and atypical findings than volleyball $(p=0.01)$ and football $(p=0.01)$.

Fig. 1 presents information about the number of samples analyzed per year in each sport. Overall, all sports tended to have an increase in the number of samples analyzed per year from 2003 to 2015. In addition, most of the sports had the largest number of samples analyzed per year in 2015 . Fig. 2 shows the changes in the percentage of AAF per year within each sport. Despite the increase in number of samples analyzed each year, there was not a clear tendency for a higher prevalence of AAF with time. In fact, some sports, such as cycling, boxing and triathlon, showed a progressive reduction in the percentage of adverse and atypical findings from 2003 to 2015.

In individual sports, the overall proportion of samples obtained in competition was $53.1 \% \pm$ $3.3 \%$, while the remaining $46.9 \% \pm 3.3 \%$ were obtained out of competition. Skiing, tennis, rowing and shooting had unexpected distributions for the samples obtained in competition and out of competition (Fig. 3A, $p<0.05$ ). In individual sports, the proportion of AAF in samples obtained in competition vs. out of competition was $67.8 \% \pm 8.7 \%$ and $32.2 \% \pm 8.7 \%$, respectively. In this case, the distributions for the AAF in completion and of competition were different from those expected in triathlon, canoeing, fencing, rowing and shooting (Fig. 3B, $p<0.05$ ). In team sports, the overall proportion of samples obtained in competition vs. out of competition was $56.3 \% \pm 2.2 \%$ and $43.7 \% \pm$ $2.2 \%$, respectively. Rugby, field hockey and football had in competition vs. out of competition distributions different from expected (Fig. 4A, $p<0.05$ ). The proportion of AAF in samples obtained in competition vs. out of competition in team sports was $71.7 \% \pm 10.9 \%$ and $28.3 \% \pm 10.9 \%$, 
respectively. Only rugby presented a distribution of adverse and atypical findings in samples in competition vs. out of competition different from what was expected (Fig. 4B, $p<0.05$ ).

\section{Discussion}

The goal of this investigation was to evaluate the number of samples analyzed and the proportion of AAF found in some of the most popular sports through the use of data on samples analyzed by WADA-accredited laboratories between 2003 and 2015. To standardise the data during this period, we used the information provided in the Testing Figures Report of 2003, which grouped all the non-negative results (i.e., AAF) in the same category. The findings in this investigation were as follows: a) Cycling, weightlifting and boxing, which had a proportion of $-3.1 \% \pm 0.7 \%$ for $\mathrm{AAF}$ during the examined period, ranked highest among the individual sports for AAF; b) ice hockey, rugby and basketball, which had a proportion of $\sim 2.1 \% \pm 0.5 \%$ for AAF during the examined period, ranked highest among the team sports for AAF; c) for most sports, the number of anti-doping controls increased yearly during the examined period, but this did not translate into a concomitant increase in the proportion of banned substances found in anti-doping controls; and d) for individual and team sports combined, the distribution of samples obtained in competition and out of competition was $55 \%$ and $45 \%$, respectively, while the distribution of AAF in competition and out of competition was $70 \%$ and $30 \%$, respectively. These results indicate that the incidence of AAF was not uniform in all sports disciplines, suggesting that some specific sports might present a greater use of banned substances. The increase in the number of anti-doping controls per year in most individual and team sports did not produce a higher percentage of AAF. In the future, focusing on samples obtained in competition might be more effective for identifying banned substances because most of the AAF were in the samples obtained in competition. This strategy might increase the strength and efficacy of anti-doping policies in those sports with the highest prevalence in the use of banned substances.

The results of the current investigation suggest that the number of samples analyzed per year is not the primary reason that there was a greater proportion of AAF in a specific sport. Recent 
research that included all the doping control test results for 13 years produced similar findings. ${ }^{12}$ Thus, both analyses provide evidence that anti-doping pressure should be focused on alternative strategies rather than on increasing the number of anti-doping controls. Table 1 indicates that the number of samples does not explain why some sports have higher percentages of AAF; thus, other causes might be responsible for the differences in the frequency of banned substances found among sports. In the current investigation, we analyzed the proportion of samples and AAF obtained in competition and out of competition within each sport, and we normalised the data to allow for a better comparison among sports. Interestingly, the individual and team sports with the highest incidence of adverse and atypical findings had a distribution of in-competition vs. out-of-competition samples comparable to the other sports analyzed. In this respect, the 2 sports with the highest proportion of in-competition samples (rowing and football) did not present particularly high percentages of AAF (Figs. 3 and 4). On the other hand, while the overall distribution of samples was comparable between in-competition and out-of-competition samples, the percentage of AAF was higher in the samples obtained in competition. All this information taken together points to the fact that the number of samples or their distribution in competition and out of competition does not explain the differences in the percentages of AAF among sports.

The explanation for the differences in the percentages of banned substances among sport disciplines found in this investigation probably includes other parameters not related to anti-doping pressure. For example, athletic success, financial gain, the assumption that other athletes also use banned substances ${ }^{23}$ and the athlete's ego, ${ }^{24}$ none of which are equal in all sports, might be behind the diverse sport-specific attitudes and beliefs toward doping misconduct. Although the reasons for the differences in the proportion of AAF among sports are not evident from the current analysis, it seems clear that doping is an uneven phenomenon of variable magnitude depending on the nature of the sports discipline. Interestingly, although the magnitude of the prevalence of doping is much higher when measured with anonymous surveys against doping control tests, the sports with the 
highest prevalence and incidence of doping are the ones that have used these 2 types of analyses. ${ }^{7,15 \text {, }}$ 17-19 The current analysis underlines the complex and idiosyncratic nature of the use of banned substances in sports, ${ }^{25}$ but it also suggests that some sports disciplines might be at a higher risk of doping misconduct. The study of the reasons for the differences in the proportion of AAF among sports warrants further investigation.

Among individual sports, there is no a clear explanation for the higher proportion of banned substances in cycling, weightlifting and boxing when compared to the remaining disciplines. From a physiological standpoint, the physical capabilities linked to success in these 3 disciplines are quite different, with uneven involvements of muscle strength, muscle power and endurance among them. Thus, one might expect that, despite the similarities in the proportions of AAF in these individual sports, the most prevalent types of banned substances found in cycling, weightlifting and boxing would be different. Although cycling was the sport with the highest incidence of AAF for the period studied, the percentage of banned substances found in the cyclists' samples clearly declined over time (Fig. 2). It might be worth studying the strategies used to fight against doping in cycling, particularly the ones set in motion by the Union Cycliste Internationale (UCI) and by WADA, along with the recommendations made by the Movement For Credible Cycling. These strategies might be useful in other sports with high percentages of AAF.

Regarding team sports, all of them share a common nature in that they require high-intensity actions intermittently combined with short periods of recovery; thus, success is driven by a reliance on anaerobic and aerobic metabolic pathways. However, the team sports with the highest proportion of AAF (ice hockey, rugby, and basketball) have similar physical demands when compared with the other team sports studies. Football was the team sport with the highest number of samples analyzed per year and with the highest proportion of samples obtained in competition. However, the percentage of AAF was low, suggesting that the use of banned substances in football is low. Finally, 
the frequency of AAF was similar in individual and team sports, probably because the factors that predispose athletes to doping are equally present in both individual and team disciplines.

Previous research has established ${ }^{3,6}$ that the most accurate way to estimate the prevalence and incidence of doping in elite sports is to use a combination of different anonymous questionnaires, specifically the survey technique called the randomised response technique. Other researchers ${ }^{4,26}$ have recommended using a combination of qualitative and quantitative measurements-interviews, questionnaires and, ideally, less-invasive biomedical tests (e.g. based on hair or salivary samples)— to assess prevalence and incidence of doping in sports. Although these 2 multidisciplinary perspectives $^{4,6}$ are useful for the assessment of attitudes toward doping in research with only a limited number of participants, their application to large populations might be very complex or even unfeasible. Additionally, an athlete's ability to correctly identify doping misconducts can affect the outcomes of these types of investigations, and those who lie about their doping behaviours still are highly influential. ${ }^{11}$ The current analysis does not contain individual information directly obtained from athletes beyond the evidence provided by the analysis of the samples, and thus it precludes, in part, a knowledge and understanding of the motives and attitudes behind doping misconduct. However, our study did provide a robust scrutiny of all the samples analyzed for anti-doping purposes from 2003 to 2015 , which strengthens the generalisability and utility of the results here presented. Given our results, it is safe to conclude that some individual sports, such as cycling, weightlifting, and boxing, and team sports, such as rugby, ice hockey and basketball, presented a statistically significant higher frequency of banned substances detected in their athletes' samples, and thus it is reasonable to assume that these sports are ones that present a greater use of prohibited substances.

As was mentioned in the Methods section, the adverse and atypical findings presented in this investigation did not necessarily end in anti-doping rule violations. Some of the substances detected might have been used for therapeutic purposes under the TUE protocol, or the AAF might not have 
resulted in a formal sanction. Since 2013, WADA has published an annual report that relates the number of adverse and atypical findings to the proportion of anti-doping rule violations. In these reports, the overall proportion of AAF that ended in an anti-doping rule violation was $64 \% \pm 1 \%$, with approximately $10 \% \pm 2 \%$ exemptions due to TUEs. Interestingly, the remaining $26 \% \pm 2 \%$ of AAF did not end in a sanction or they were still pending of a final decision which might affect the final number of AAF that ends in a sanction. The translation from adverse and atypical finding to anti-doping rule violations might also depend on the sport discipline. For example, from 2013 to $2015,85.5 \% \pm 4.6 \%$ and $84.8 \% \pm 1.5 \%$ of the AAF found in wrestling and weightlifting, respectively, finally ended in antidoping rule violations, while this proportion was only $23.3 \% \pm$ $14.3 \%$ in gymnastics. In team sports, the sport with the highest conversion of AAF into anti-doping rule violations was rugby $(69.8 \% \pm 5.5 \%)$ followed by volleyball $(69.3 \% \pm 23.7 \%)$. Thus, the data presented in this investigation are useful in assessing the use of banned substances in different sports, but the relationship between the number of AAF and the number of anti-doping rule violations among various sports requires further analysis.

The current analysis has some limitations that should be considered when drawing conclusions about the use of banned substances in these sports. First, the Testing Figures Reports made public by WADA do not include information about the athlete's nationality even though this characteristic might affect the occurrence of misconduct regarding doping. ${ }^{14}$ The reports provided by WADA include information about the number of samples analyzed and the percentage of AAF in each WADA-accredited laboratory. However, this information cannot be extrapolated to infer the nationalities of the athletes whose samples were analyzed in each laboratory or the relationship between the athletes' nationalities and the prevalence of AAF. By including the athletes' nationalities in its reports, WADA could help us understand the nature of doping in various countries, especially after the recent independent investigations into doping practices that implicated Russian athletes, coaches, doctors, and the accredited laboratory based in Moscow. ${ }^{27}$ Second, WADA's reports do not 
include any analyses or information that allows us to differentiate between the occurrence of AAF and the sex of athletes. The sex of athletes might affect the prevalence and incidence of AAF, specifically as they relate to the concentration of hormones such as testosterone and to conditions in which androgens are elevated, such as disorders of sex development (DSD). ${ }^{28}$ The use of sex-specific information might help to identify discrepancies in the occurrence of doping misconduct among male and female athletes, but at present this analysis cannot be inferred from the WADA's reports. Finally, WADA's reports only include information on samples analyzed and substances found, but the use of banned substances in sports may be greater than recognized because not all athletes are under antidoping scrutiny and the methodologies used in the analyses are imperfect. Despite these limitations, the current analysis presents a broad vision of the frequency of AAF in sports.

\section{Conclusion}

In summary, the incidence of AAF reported by WADA-accredited laboratories was not uniform in all sports disciplines from 2003 to 2015. While some sports had a proportion of AAF above $3 \%$, other disciplines did not even reach $1 \%$. Thus, despite WADA's proposal of a concerted fight against doping, those who deyelop anti-doping strategies should consider the differences in the risk of doping among sports. Overall, the number of anti-doping controls has increased yearly in most sports, but this has not translated into a concomitant increase in the proportion of banned substances used in sports, except for cycling. Given the disproportionate distribution of AAF in competition and out of competition, more pressure should be exerted, in general terms, to obtain samples in competition, although this strategy should be designed specifically for each sport in the light of the current data. This information may be valuable to national and international anti-doping organisations in their efforts to improve the policies used to reduce doping practices in sports and to identify specific sports disciplines that have a higher risk of doping misconduct. 


\section{Acknowledgments}

The current investigation has been possible thanks to WADA, which makes its statistics from its accredited anti-doping laboratories public. Thus, we want to acknowledge the labour of WADA in making its statistics publicly available and in implementing other policies in the fight against doping in sports. This study did not receive any funding.

\section{Authors's contributions}

MAN, JMG, MMP and JDC conceived the idea of the investigation; MAN extracted the data; MAN and JDC performed the statistical analysis and drafted the manuscript; JMG and MMP critically reviewed the manuscript.

\section{Competing Interests}

The authors declare that they have no competing interests. 


\section{REFERENCES}

(1) Zhang Z. Establishing an anti-doping internal whistleblower policy in China. J Sport Health Sci 2018;7:337-8.

(2) Dvorak J, Saugy M, Pitsiladis YP. Challenges and threats to implementing the fight against doping in sport. Br J Sports Med 2014;48:807-9.

(3) Pielke R Jr. Assessing doping prevalence is possible. So what are we waiting for? Sports Med 2017;48:207-9.

(4) Morente-Sánchez J, Zabala M. Doping in sport: a review of elite athletes' attitudes, beliefs, and knowledge. Sports Med 2013;43:395-411.

(5) Herzog W. Fairness in Olympic sports: how can we control the increasing complexity of doping use in high performance sports? J Sport Health Sci 2017;6:47.

(6) de Hon O, Kuipers H, van Bottenburg M. Prevalence of doping use in elite sports: a review of numbers and methods. Sports Med 2015;45:57-69.

(7) Ulrich R, Pope HG Jr, Cleret L, Petroczi A, Nepusz T, Schaffer J, et al. Doping in two elite athletics competitions assessed by randomized-response surveys. Sports Med 2017;48:211-9.

(8) Pitsch W, Emrich E, Klein M. Doping in elite sports in Germany: results of a www survey. Eur J Sport Soc 2007;4:89-102.

(9) Frenger M, Pitsch W, Emrich E. Sport-induced substance use-an empirical study to the extent within a German sports association. PLoS One 2016;11:e0165103. doi: 10.1371/journal.pone.0165103.

(10) Petroczi A, Aidman EV, Hussain I, Deshmukh N, Nepusz T, Uvacsek M, et al. Virtue or pretense? Looking behind self-declared innocence in doping. PLoS One 2010;5:e10457. doi: 10.1371/journal.pone.0010457.

(11) Schroter H, Studzinski B, Dietz P, Ulrich R, Striegel H, Simon P. A comparison of the Cheater Detection and the unrelated question models: A randomized response survey on 
physical hysical and doping in recreational . PLoS One 2016;11:e0155765. doi: 10.1371/journal.pone.0155765.

(12) Aguilar M, Munoz-Guerra J, Plata MDM, Del Coso J. Thirteen years of the fight against doping in figures. Drug Test Anal 2017;9:866-9.

(13) Ferro P, Ventura R, Pérez-Mañá C, Farré M, Segura J. Genetic and protein biomarkers in blood for the improved detection of GH abuse. J Pharm Biomed Anal 2016;128:111-8.

(14) Sottas P-E, Robinson N, Fischetto G, Dollé G, Alonso JM, Saugy M. Prevalence of blood doping in samples collected from elite track and field athletes. Clin chem 2011;57:762-9.

(15) Loraschi A, Galli N, Cosentino M. Dietary supplement and drug use and doping knowledge and attitudes in Italian young elite cyclists. Clin J Sport Med 2014;24:238-44.

(16) Morente-Sanchez J, Mateo-March M, Zabala M. Attitudes towards doping and related experience in Spanish national cycling teams according to different Olympic disciplines. PLoS One 2013;8:e70999. doi: 10.1371/journal.pone.0070999.

(17) Baume N, Jan N, Emery C, Mandanis B, Schweizer C, Giraud S, et al. Antidoping programme and biological monitoring before and during the 2014 FIFA World Cup Brazil. $\mathrm{Br}$ J Sports Med 2015;49:614-22.

(18) Alaranta A, Alaranta H, Holmila J, Palmu P, Pietila K, Helenius I. Self-reported attitudes of elite athletes towards doping: differences between type of sport. Int $J$ Sports Med 2006;27:842-6.

(19) Lazuras L, Barkoukis V, Rodafinos A, Tzorbatzoudis H. Predictors of doping intentions in elite-level athletes: a social cognition approach. J Sport Exerc Psychol 2010;32:694-710.

(20) Mottram D, Chester N, Atkinson G, Goode D. Athletes' knowledge and views on OTC medication. Int J Sports Med 2008;29:851-5.

(21) World Anti-Doping Agency. Anti-Doping Testing Figures; 2017.Available at: https://www.wada-ama.org/en/resources/laboratories/anti-doping-testing-figures-report [accessed 01.05.2019] 
(22) Boutilier MA, Giovanni LFS, University SH. Individual and team in the Olympics: A question of balance. Int Rev Sociol Sport 1992;27:177-89.

(23) Birzniece V. Doping in sport: effects, harm and misconceptions. Intern Med J 2015;45:23948.

(24) Ring C, Kavussanu M. Ego involvement increases doping likelihood. J Sports Sci 2018;36:1757-62.

(25) Whitaker L, Backhouse S. Doping in sport: an analysis of sanctioned UK rugby union players between 2009 and 2015. J Sports Sci 2017;35:1607-13.

(26) Morente-Sanchez J, Zabala M. Knowledge, attitudes and beliefs of technical staff towards doping in Spanish football. J Sports Sci 2015;33:1267-75.

(27) World Anti-Doping Agency. The Independent Commission Report \#1; 2015. Available at: https://www.wada-

ama.org/sites/default/files/resources/files/wada_independent_commission_report_1_en.pdf. [accessed 05.11.2018].

(28) Clark RV, Wald JA, Swerdloff RS, Wang C, Wu FCW, Bowers LD, et al. Large divergence in testosterone concentrations between men and women: frame of reference for elite athletes in sex-specific competition in sports, a narrative review. Clin Endocrinol (Oxf) 2018. 


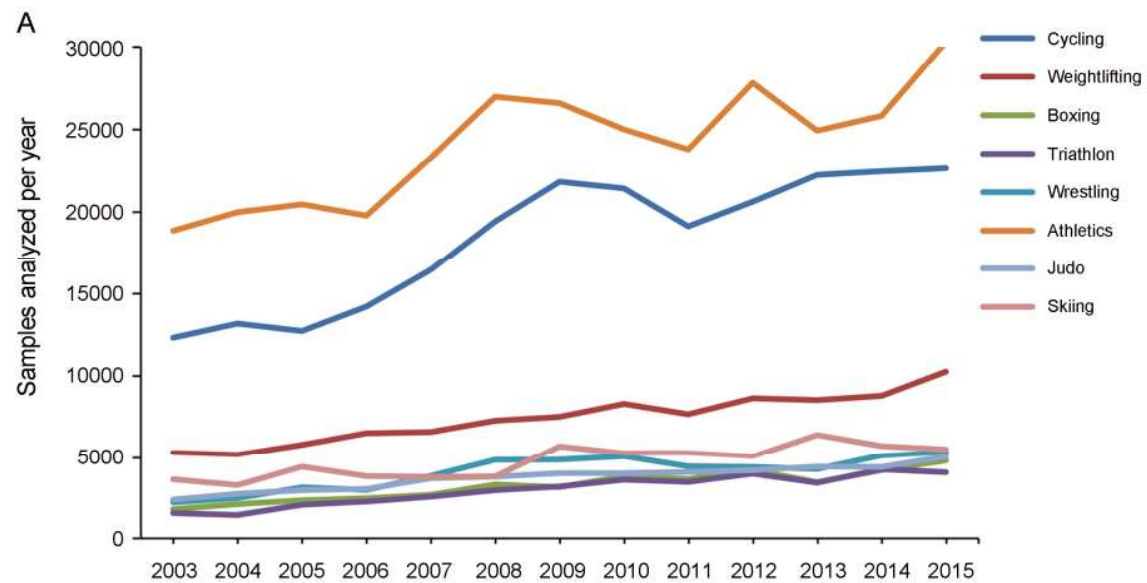

B

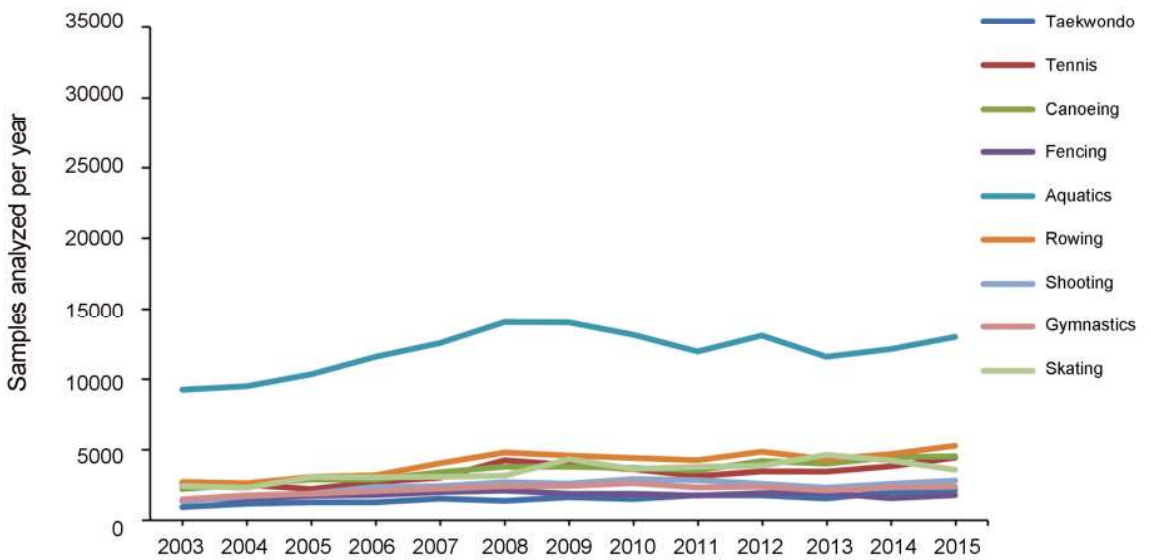

C

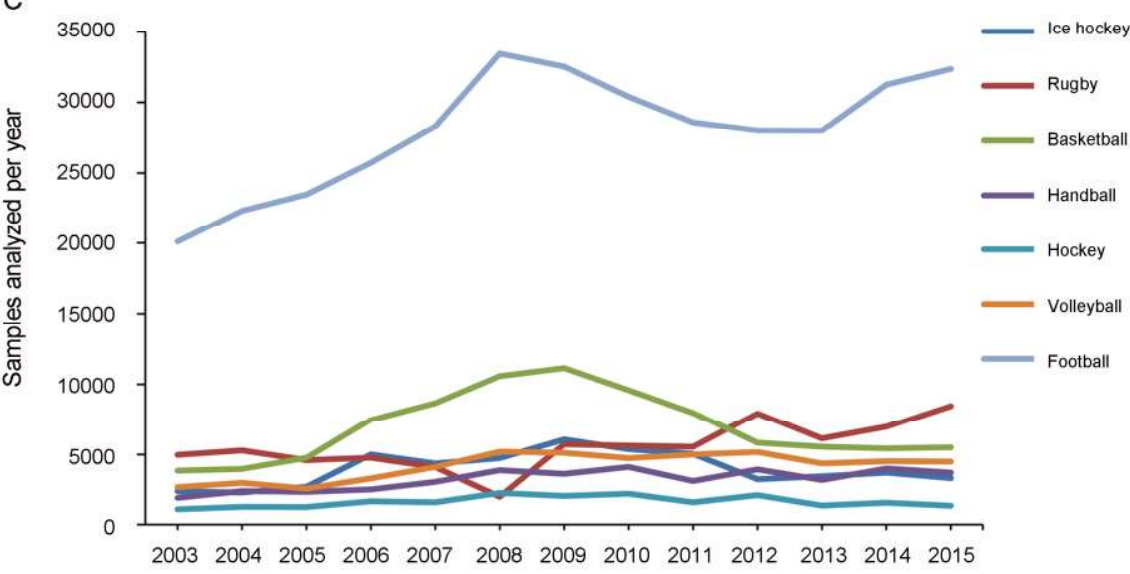

Fig. 1 Number of samples analyzed per year between 2003 and 2015 in individual sports (A and B) and team sports $(\mathrm{C})$. 


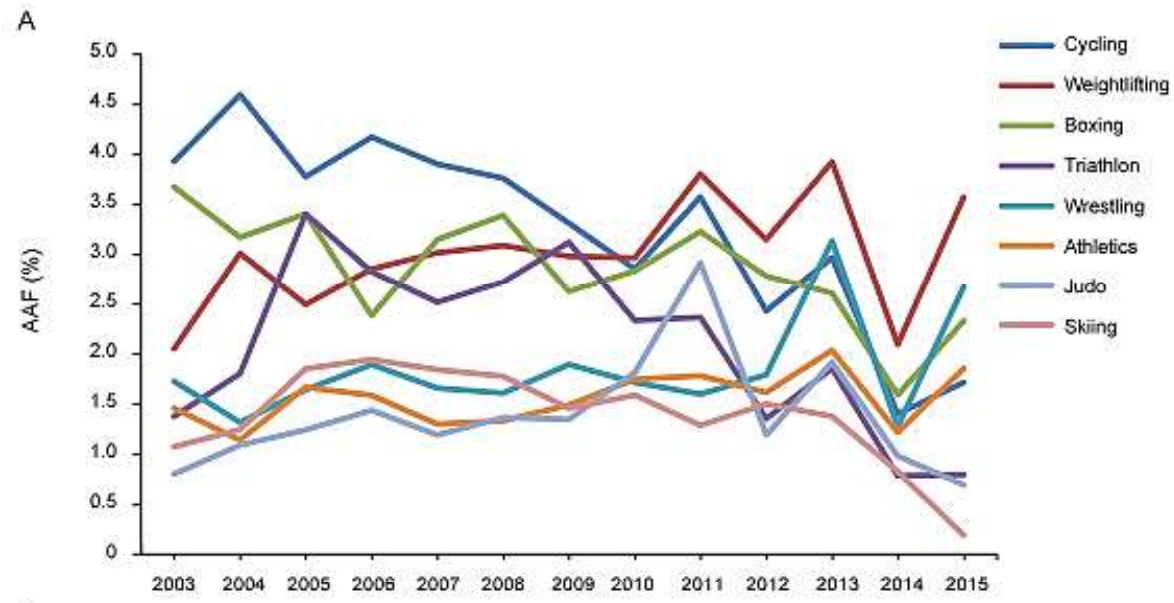

B

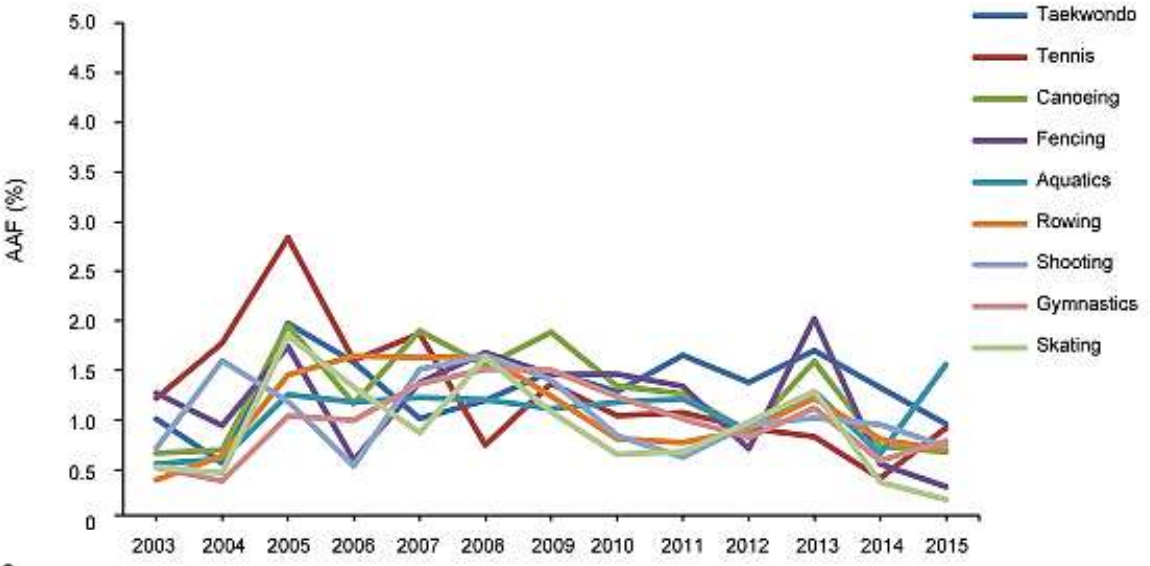

$\mathrm{C}$

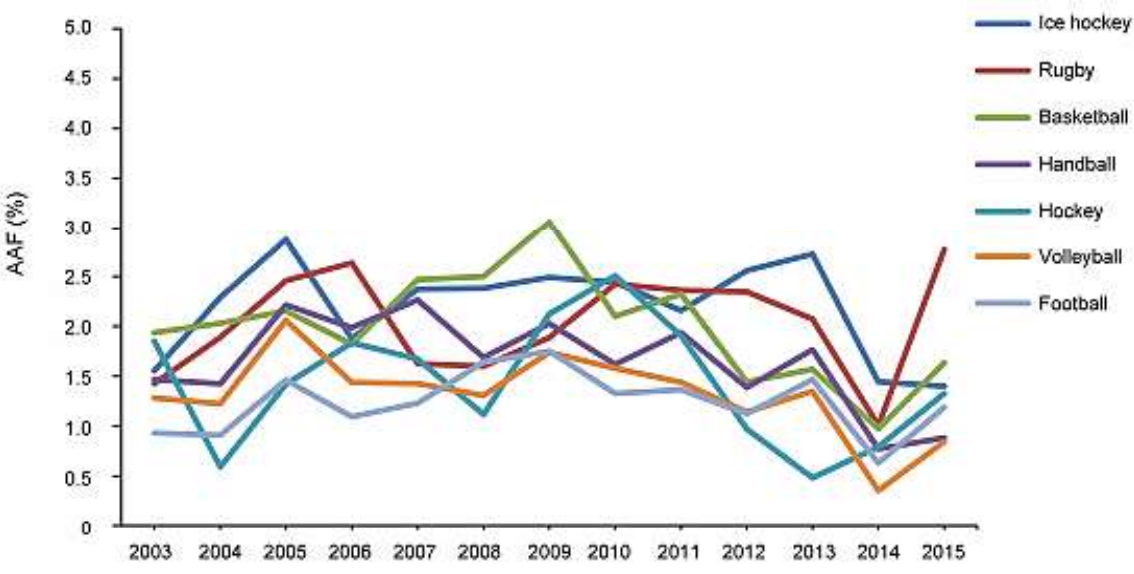

Fig. 2 Percentage of adverse and atypical findings (AAFs) per year between 2003 and 2015 in individual sports (A and B) and team sports (C). 


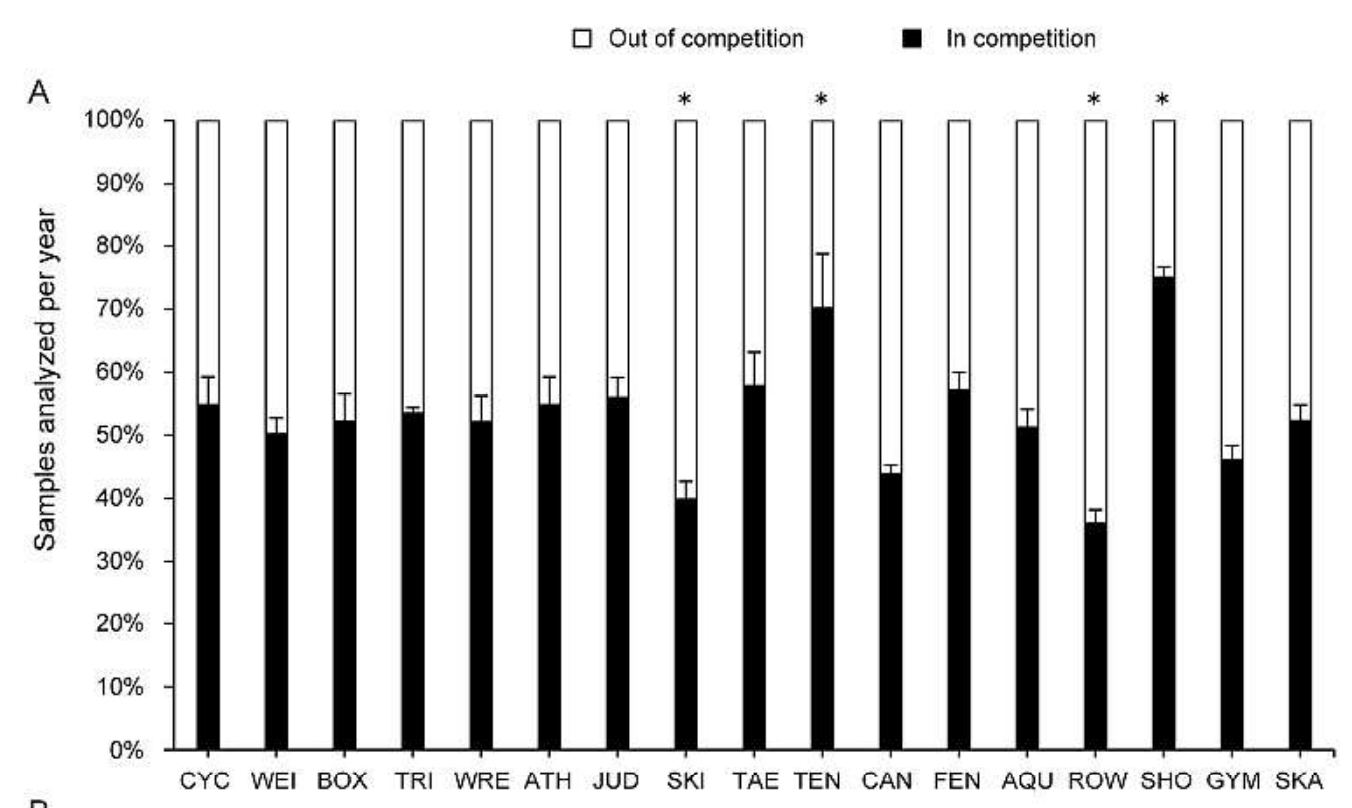

B

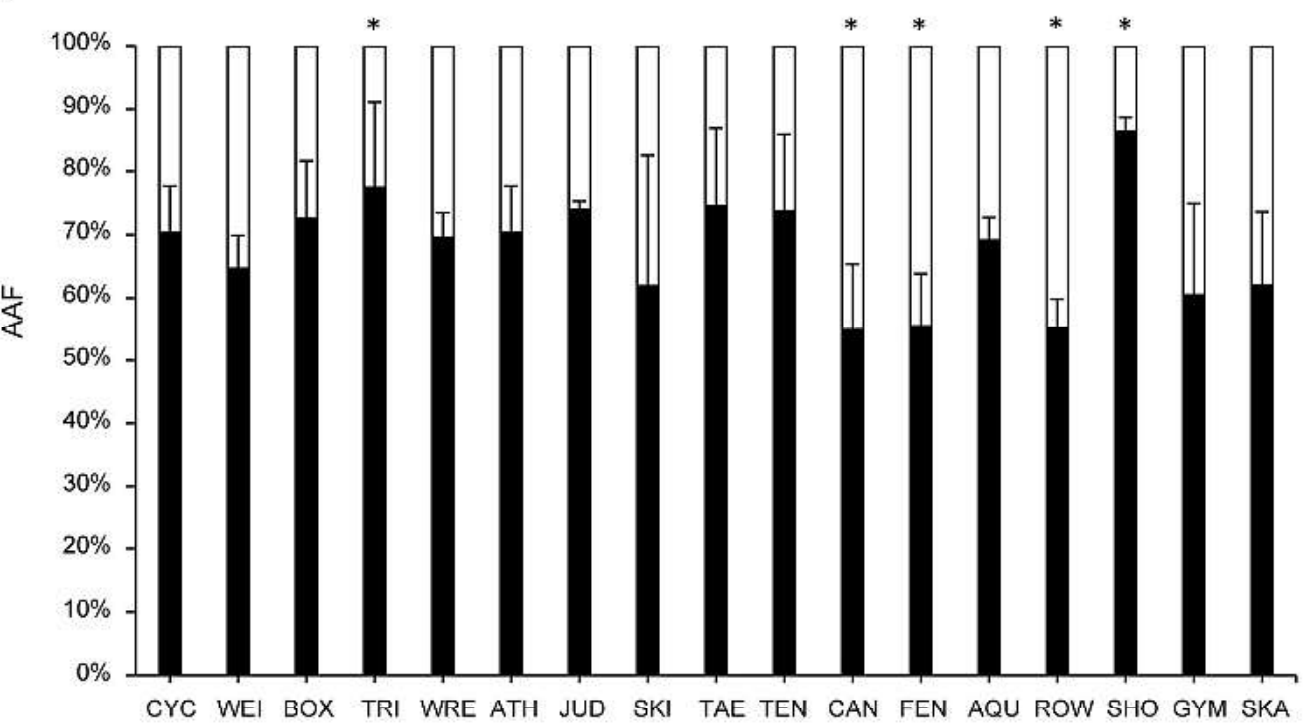

Fig. 3 (A) Proportion of samples obtained in competition and out of competition in individual sports and (B) distribution of the AAF in samples obtained in competition and out of competition. The data are mean \pm SD for each sport between 2003 and 2015. * The distribution was different from that expected at $p<0.05 . \mathrm{AAF}=$ adverse and atypical finding; $\mathrm{AQU}=$ Aquatics; $\mathrm{ATH}=$ Athletics; $\mathrm{BOX}$ $=$ Boxing; $\mathrm{CAN}=$ Canoe/kayaking $; \mathrm{CYC}=$ Cycling $; \mathrm{FEN}=$ Fencing; $\mathrm{GYM}=$ Gymnastics; $\mathrm{JUD}=$ Judo; ROW=Rowing; SHO = Shooting; SKA = Skating; SKI = Skiing; TAE = Taekwondo; TEN = Tennis; TRI $=$ Triathlon; WEI $=$ Weightlifting; $\mathrm{WRE}=$ Wrestling. 


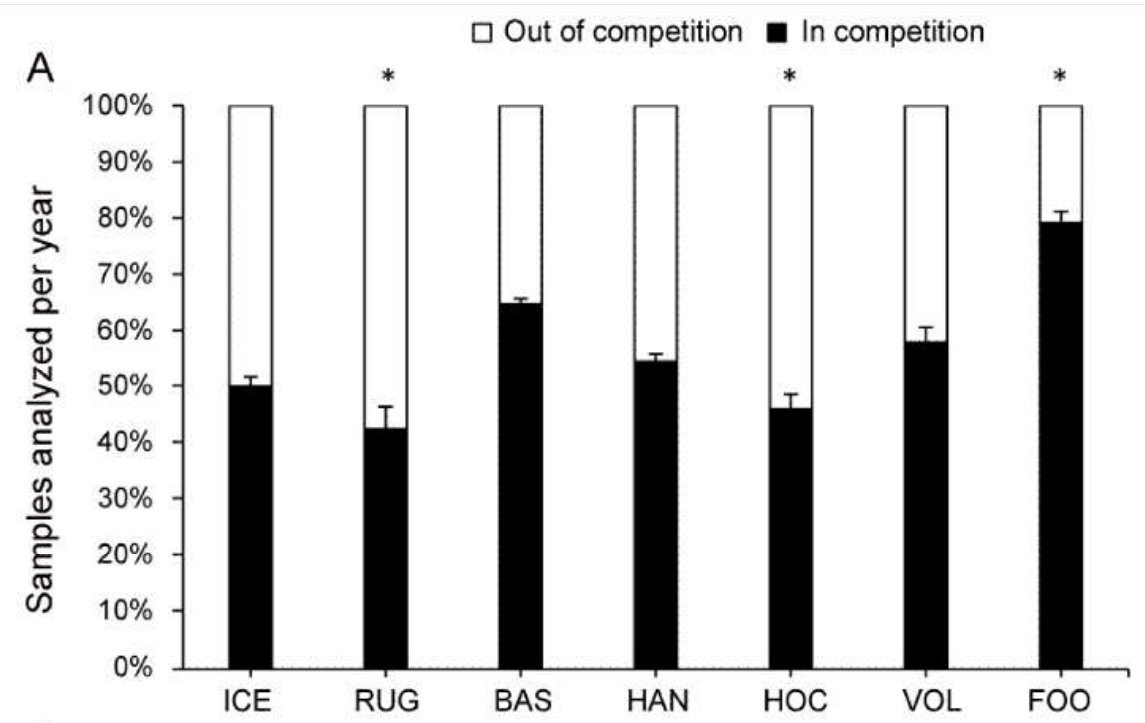

B

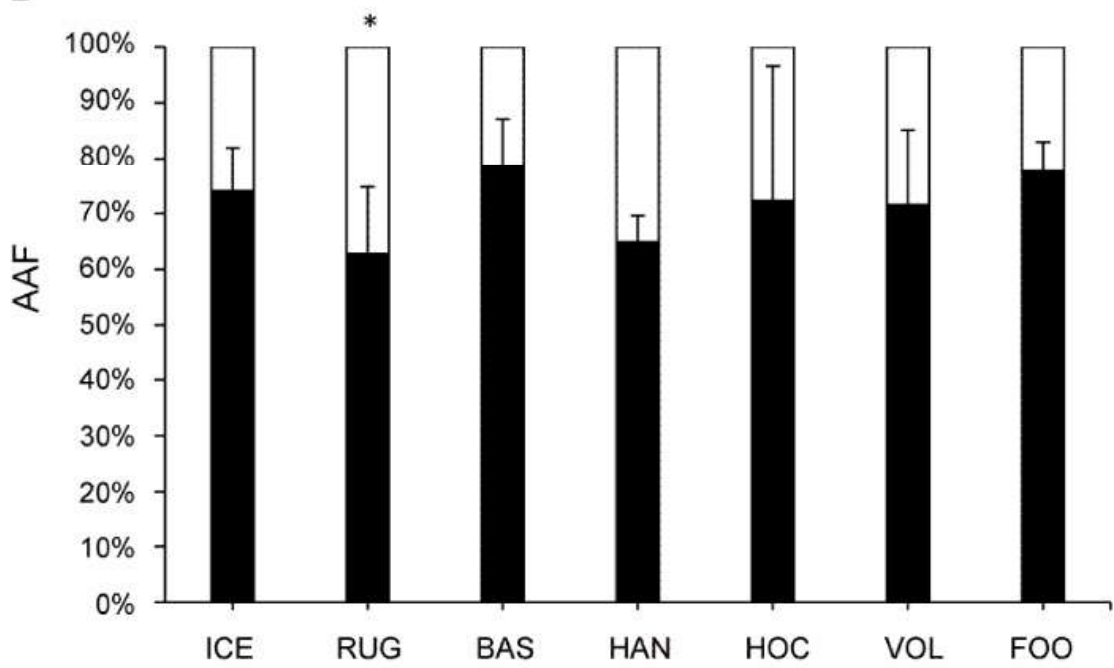

Fig. 4 (A) Proportion of samples obtained in competition and out of competition in team sports and (B) distribution of the AAF in samples obtained in competition and out of competition. The data are mean \pm SD for each sport between 2003 and 2015. * The distribution was different from that expected at $p<0.05 . \mathrm{AAF}=$ adverse and atypical finding; $\mathrm{BAS}=$ Basketball; $\mathrm{FOO}=$ Football; HAN = Handball; HOC ₹ Field Hockey; ICE = Ice Hockey; RUG = Rugby; VOL = Volleyball. 
Table 1 Number of samples analyzed, number and percentage of AAF in individual and team sports between 2003 and $2015($ mean \pm SD)

\begin{tabular}{lccc}
\hline Individual sport & Samples (number) & AAF (number) & AAF (\%) \\
\hline Individual sport & & & \\
Cycling & $18371 \pm 4018$ & $572 \pm 128$ & $3.3 \pm 1.0$ \\
Weightlifting & $7440 \pm 1491^{\$}$ & $227 \pm 75$ & $3.0 \pm 0.6$ \\
Boxing & $3303 \pm 892^{*}$ & $92 \pm 21$ & $2.9 \pm 0.6$ \\
Triathlon & $3069 \pm 921^{*}$ & $61 \pm 25$ & $2.1 \pm 0.8^{* \dagger}$ \\
Wrestling & $4136 \pm 1032^{*}$ & $78 \pm 33$ & $1.8 \pm 0.5^{* \dagger}$ \\
Athletics & $24132 \pm 3516^{\$}$ & $379 \pm 96$ & $1.6 \pm 0.3^{* \dagger}$ \\
Judo & $3820 \pm 752^{*}$ & $54 \pm 27$ & $1.4 \pm 0.6^{* \dagger}$ \\
Skiing & $4801 \pm 989^{*}$ & $65 \pm 23$ & $1.4 \pm 0.5^{* \dagger}$ \\
Taekwondo & $1579 \pm 332^{*}$ & $21 \pm 7$ & $1.3 \pm 0.5^{* \dagger}$ \\
Tennis & $3345 \pm 686^{*}$ & $40 \pm 13$ & $1.3 \pm 0.6^{* \dagger}$ \\
Canoe/kayaking & $3559 \pm 717^{*}$ & $45 \pm 18$ & $1.3 \pm 0.5^{* \dagger}$ \\
Fencing & $1836 \pm 187^{*}$ & $22 \pm 11$ & $1.2 \pm 0.5^{* \dagger}$ \\
Aquatics & $12010 \pm 1539^{\$}$ & $131 \pm 44$ & $1.1 \pm 0.3^{* \dagger}$ \\
Rowing & $4082 \pm 865^{*}$ & $44 \pm 19$ & $1.1 \pm 0.4^{* \dagger}$ \\
Shooting & $2427 \pm 469^{*}$ & $26 \pm 26$ & $1.1 \pm 0.4^{* \dagger}$ \\
Gymnastics & $2224 \pm 316^{*}$ & $23 \pm 10$ & $1.0 \pm 0.4^{* \dagger}$ \\
Skating & $3496 \pm 702^{*}$ & $32 \pm 18$ & $0.9 \pm 0.5$ \\
Team sport & & & \\
Ice Hockey & $4004 \pm 1187$ & $89 \pm 34$ & $2.2 \pm 0.5$ \\
Rugby & $5555 \pm 1646$ & $115 \pm 53$ & $2.0 \pm 0.5$ \\
Basketball & $6951 \pm 2457^{*}$ & $147 \pm 87$ & $2.0 \pm 0.5$ \\
Handball & $3255 \pm 720^{\#}$ & $53 \pm 16$ & $1.6 \pm 0.5$ \\
FieldHockey & $1723 \pm 383^{\S} *$ & $25 \pm 14$ & $1.4 \pm 0.6^{\& \S}$ \\
Volleyball & $4194 \pm 948^{\#}$ & $55 \pm 20$ & $1.3 \pm 0.4^{*} \S$ \\
Football & $28039 \pm 4143^{\$}$ & $352 \pm 121$ & $1.2 \pm 0.3^{*} \S$ \\
\hline
\end{tabular}

* Different from cycling; ${ }^{\dagger}$ Different from weightlifting; ${ }^{\dagger}$ Different from boxing; ${ }^{\#}$ Different from basketball; ${ }^{\&}$ Different from ice hockey; ${ }^{\S}$ Different from rugby; ${ }^{\$}$ Different from all the remaining sports within the same category.

Abbrevation: $\mathrm{AAF}=$ adverse and atypical finding. 